\title{
Co-relation Between Perinatal Asphyxia with Hypoxic Ischemic Encephalopathy (PNA with HIE) and Blood Sugar Level
}

\author{
Abdul Hakim ${ }^{1}$, Mahmuder Rahman ${ }^{2}$, Morium Begum ${ }^{3}$ \\ ${ }^{1}$ Department of Pediatrics, Rangpur Medical College, Rangpur, Bangladesh \\ ${ }^{2}$ Department of Pediatrics, Faridpur Medical College, Faridpur, Bangladesh \\ ${ }^{3}$ Department of Obstetrics \& Gynaecology, Rangpur Medical College Hospital, Rangpur, Bangladesh
}

Email address:

drhakim67@yahoo.com (A. Hakim)

\section{To cite this article:}

Abdul Hakim, Mahmuder Rahman, Morium Begum. Co-relation Between Perinatal Asphyxia with Hypoxic Ischemic Encephalopathy (PNA with HIE) and Blood Sugar Level. American Journal of Biomedical and Life Sciences. Vol. 9, No. 5, 2021, pp. 267-270.

doi: 10.11648/j.ajbls.20210905.17

Received: March 4, 2021; Accepted: August 2, 2021; Published: October 28, 2021

\begin{abstract}
Introduction: Perinatal asphyxia is one of the most common primary causes of mortality and morbidity among neonates. Perinatal asphyxia will result in neonatal hypoxia and tissue/organ injury. Objective: The aim of this study was to observe the Co-relation between Perinatal asphyxia with Hypoxic Ischemic Encephalopathy (PNA with HIE) and Blood sugar level. Methodology: This is a descriptive cross-sectional case study where a total of 182 neonates were admitted to the hospital with the problem of Perinatal asphyxia with Hypoxic Ischemic Encephalopathy and with some other associated problem. Result: Among the cases, 114 (62.6\%) were Boys and 68 (37.45\%) Girls. The mean age was 29.72 \pm 78.42 , Weight 2.77 \pm .60 , RBS $6.57 \pm 7.38$. Among the asphyxiated neonates blood sugar levels in arterial cord blood were significantly lower and had a negative $(\mathrm{r}=-0.195, \mathrm{P}<0.01)$ correlation with the severity of birth asphyxia and different stages of HIE. Conclusion: Perinatal asphyxia can result in neonatal hypoxia and tissue/organ injury. A variety of maternal, obstetric, and neonatal conditions predispose the fetus and newborn to asphyxia, which can occur before, during, or after birth From our result, we can conclude that the relationship between Perinatal asphyxia and blood sugar level is significantly lower and negative.
\end{abstract}

Keywords: Perinatal Asphyxia, Neonatal Hypoxia, Organ Injury

\section{Introduction}

Perinatal asphyxia is globally recognized as the fifthlargest cause of under-five child deaths $(8.5 \%)$, following pneumonia, diarrhea, neonatal infections, and complications of preterm birth. Internationally, almost 5 million neonates are diagnosed with birth asphyxia every year [1]. According to the World Health Organization (WHO) definition, failure to initiate and sustain breathing at birth is categorized as perinatal asphyxia. Glucose is the main source of energy for the human brain which provides up to $90 \%$ of the brain energy supply under normal conditions. As infants have a limited ability to generate alternate substrates, maintenance of an adequate supply of glucose to the brain is extremely important. Disturbances in glucose and oxygen supply to the human fetus at the time of labor and delivery may cause perinatal asphyxia, along with subsequent hypoxic-ischemic encephalopathy (HIE). Extremely low glucose levels can often cause encephalopathy in patients, which can then lead to long-term neurological ailments. During birth asphyxia, various complications like severe glycogen depletion, catecholamine release, and idiopathic hyperinsulinemia can occur, which can lead to hypoglycemia [2]. Early diagnosis is extremely challenging in neonatal care, and hypoxia and hypoglycemia can lead to profound brain damage. The early outcome of birth asphyxia is either neonatal death or the presence of hypoxic-ischemic encephalopathy (HIE). Hypoxic-ischemic brain damage is one of the more challenging consequences of perinatal asphyxia. Brief hypoxia can lead to an increase in lactate, fall in $\mathrm{pH}$, decrease in high-energy phosphate compounds by impairing the cerebral oxidative metabolism, due to anaerobic glycolysis to 
generate ATP [1]. During anaerobic conditions, one molecule of glucose yields only two molecules of ATP, as compared to 38 molecules during aerobic conditions [3]. Glucose is rapidly metabolized anaerobically during asphyxia, which helps in minimizing cellular energy depletion in tissues of the brain and other areas [4]. Glucose is an essential energy supplier in a developing brain and oxidative metabolism accounts for almost all of the glucose uptake by the brain [5]. The hypoxic brain, therefore, increases its glucose utilization. It is common knowledge that blood is the major source of brain glucose. A drop in the blood glucose concentration level increases the risk of brain injury. Hypoxia can cause vasodilatation, which increases the glucose availability for anaerobic glycolysis. This in turn helps increase the lactic acid production of the body. Decreased glycolysis, loss of cerebrovascular autoregulation, and weakened cardiac function are associated with the worsening of acidosis. These factors can then cause local ischemia and decreased glucose delivery to the tissues that have increased its substrate utilization. Increased glycolytic substrates, distal to the phosphofructokinase step, from the state, are absent in hypoglycemia [6]. Because of this, local glucose storage becomes depleted and results in a fall in energy reserves and accumulation of lactic acid. A reduction in the blood glucose level and oxygen supply can lead to failure in ATP generation and culminates in cellular damage. The increased excitotoxic cellular damage and lack of ATP can lead to ADP and AMP, which are converted into adenosine, inosine, and hypoxanthine. In uninterrupted tissue hypoxia with reperfusion, hypoxanthine is oxidized into xanthine and uric acid in the presence of xanthine oxidase. This causes an increase in the uric acid level in the blood [6]. During the reoxygenation period following the initial phase of asphyxia, oxygen-derived free radicals are produced along with uric acid formation, which can be linked to the severity of perinatal asphyxia [6]. The increased uric acid production can draw a parallel among the generation of vicious cycles of tissue damage, oxygen-free radicals, ADP and AMP generation, fall in ATP generation, and the decrease of glucose in the body. Few studies have been conducted on how blood glucose levels contribute to the severity of asphyxia and encephalopathy $[7,8]$. The primary goal of this study is to find the relation of hypoglycemia $(<40 \mathrm{mg} / \mathrm{dL})$ in the development of brain injury in asphyxiated newborns.

\section{Objective}

To identify the Co-relation between Perinatal asphyxia with Hypoxic Ischemic Encephalopathy (PNA with HIE) and Blood sugar level.

\section{Methodology}

This was a descriptive cross-sectional case study where a total of 182 neonates were admitted in the hospital who were suffering perinatal asphyxia with Hypoxic Ischemic Encephalopathy and with some other associated problem. A purposive sampling technique was used to select the study participants. Neonates were having perinatal asphyxia with Hypoxic Ischemic Encephalopathy stage 1 to 3 with some other associated problems. Detailed clinical and neurological examination was done for all the neonates included in the study. Cases were classified into mild, moderate, or severe hypoxic-ischemic encephalopathy (HIE) following other studies. Descriptive and inferential statistical analysis has been carried out in the present study. Significance was assessed at a $5 \%$ level of CI. Bivariate distribution analysis was used to determine the correlations (two-tailed Karl Pearson's coefficient of correlation, indicated as r) between Perinatal asphyxia with Hypoxic Ischemic Encephalopathy (PNA with HIE) and Blood sugar level. The statistical data analysis was done using SPSS software version 23.0 for Windows.

Inclusion criteria:

1) Both boy and girl babies having the problem of perinatal asphyxia with Hypoxic Ischemic Encephalopathy and with some other associated problem.

2) Major polytrauma and pathological fractures are excluded from this study.

3) Full term neonates aged between 0.5 hours to 960 hours.

4) Birth weight between $1.5-5 \mathrm{~kg}$.

Data collection:

All the findings were collected from the case study.

\section{Result}

Among the cases 114 (62.6\%) were Boys and 68 (37.45\%) Girls. The mean age was $29.72 \pm 78.42$, Weight $2.77 \pm .60$, RBS $6.57 \pm 7.38$.

Table 1. Gender Distribution of the patients. $(n=182)$.

\begin{tabular}{lll}
\hline Gender & N & \% \\
\hline Boy & 114 & 62.6 \\
Girl & 68 & 37.4 \\
Total & 182 & 100 \\
\hline
\end{tabular}

Table 2. Mean and Standard Deviation of Numeric Variables. $(n=182)$.

\begin{tabular}{ll}
\hline Variables & Mean \pm SD \\
\hline Age & $29.72 \pm 78.42$ \\
Weight & $2.77 \pm .60$ \\
RBS-mmol/L & $6.57 \pm 7.38$ \\
\hline
\end{tabular}

Among the asphyxiated neonates a number of 93 (51.1\%) developed Perinatal asphyxia with Hypoxic Ischemic Encephalopathy stage 1, $85(46.7 \%)$ developed Perinatal asphyxia with Hypoxic Ischemic Encephalopathy stage 2, and $3(1.6 \%)$ developed Perinatal asphyxia with Hypoxic Ischemic Encephalopathy stage 3. Among the asphyxiated neonates $64(56.1 \%)$ boy and $29(42.6 \%)$ girl baby are having Perinatal asphyxia with Hypoxic Ischemic Encephalopathy stage 1, $49(43.0 \%)$ boy and $85(46.7 \%)$ girl baby are having Perinatal asphyxia with Hypoxic Ischemic Encephalopathy stage 2 and followed by. 
Table 3. Problem * Gender Cross tabulation.

\begin{tabular}{|c|c|c|c|c|}
\hline \multirow{2}{*}{ Problem } & & \multicolumn{2}{|l|}{ Gender } & \multirow{2}{*}{ Total } \\
\hline & & Boy & Girl & \\
\hline \multirow{3}{*}{ PNA-S-1 } & Count & 64 & 29 & 93 \\
\hline & $\%$ within Problem & $68.8 \%$ & $31.2 \%$ & $100.0 \%$ \\
\hline & $\%$ within Gender & $56.1 \%$ & $42.6 \%$ & $51.1 \%$ \\
\hline \multirow{3}{*}{ PNA-S-11 } & Count & 1 & 0 & 1 \\
\hline & $\%$ within Problem & $100.0 \%$ & $0.0 \%$ & $100.0 \%$ \\
\hline & $\%$ within Gender & $0.9 \%$ & $0.0 \%$ & $0.5 \%$ \\
\hline \multirow{3}{*}{ PNA-S-2 } & Count & 49 & 36 & 85 \\
\hline & $\%$ within Problem & $57.6 \%$ & $42.4 \%$ & $100.0 \%$ \\
\hline & $\%$ within Gender & $43.0 \%$ & $52.9 \%$ & $46.7 \%$ \\
\hline \multirow{3}{*}{ PNA-S-3 } & Count & 0 & 3 & 3 \\
\hline & $\%$ within Problem & $0.0 \%$ & $100.0 \%$ & $100.0 \%$ \\
\hline & $\%$ within Gender & $0.0 \%$ & $4.4 \%$ & $1.6 \%$ \\
\hline \multirow{3}{*}{ Total } & Count & 114 & 68 & 182 \\
\hline & $\%$ within Problem & $62.6 \%$ & $37.4 \%$ & $100.0 \%$ \\
\hline & $\%$ within Gender & $100.0 \%$ & $100.0 \%$ & $100.0 \%$ \\
\hline
\end{tabular}

Among the asphyxiated neonates blood suger levels in arterial cord blood was significantly lower and has a negative $(\mathrm{r}=-0.195, \mathrm{P}<0.01)$ correlation with the severity of birth asphyxia and different stages of HIE.

Table 4. Correlations between PNA and Blood sugar level.

\begin{tabular}{llll}
\hline & & Problem & RBS-mmol/L \\
\hline \multirow{3}{*}{ Problem } & Pearson Correlation & 1 & $-.195^{* *}$ \\
& Sig. (2-tailed) & & .008 \\
& $\mathrm{~N}$ & 182 & 182 \\
$\mathrm{RBS}-$ & Pearson Correlation & $-.195^{* *}$ & 1 \\
$\mathrm{mmol} / \mathrm{L}$ & Sig. (2-tailed) & .008 & \\
& $\mathrm{~N}$ & 182 & 182 \\
\hline
\end{tabular}

**. Correlation is significant at the 0.01 level (2-tailed).

\section{Discussion}

Perinatal asphyxia can potentially cause permanent damage and even death of the fetus or the newborn infant. Because of these reasons, perinatal asphyxia is recognized worldwide as a devastating clinical condition. The Apgar score plays some role in predicting the immediate outcome of asphyxia, such as $\mathrm{HIE}$ and the long-term sequelae. The potential contribution of hypoglycemia in the development of neonatal brain injury has been the subject of many research works [5]. Studies in animal models are also available [6]. An immature neonatal brain, as compared to an adult mature brain, is more resistant to hypoglycemic injury [5, 6, 9]. In our study, the cases were classified into mild, moderate, or severe hypoxic-ischemic encephalopathy (HIE), according to previous studies [9]. It was found that by lowering the cerebral energy requirements, increasing the cerebral blood flow and glucose extraction, and enhancing the ability to utilize lactate as an alternative energy source, the neonatal brain can cope with hypoglycemia [10, 11]. But when hypoglycemia develops concomitantly with asphyxia $62.6 \%$, all of the compensatory mechanisms fail to save the neonatal brain from severe sustained hypoxic damage [11]. Perinatal asphyxia primarily leads to tissue damage, mainly brain tissue damage, followed by electrolyte imbalance. Serum electrolyte status and glucose levels can help determine the extent of tissue image and the severity of asphyxia [14]. In their study, Aundhakar reported that out of 50 cases admitted to NICU, males were more prone to asphyxia.[15] In our present study, it is also showed that that males were more prone to asphyxia. The results of the present study were in concordance with those of a study by Basu, where it was reported that the asphyxiated babies developed hypoglycemia proportional to the rise in their serum uric acid level, degree of asphyxia, and severity of HIE [13]. These findings established the fact that hypoglycemia is proportional to tissue damage and adverse neurological outcomes. Hypoglycemia in perinatal asphyxia is demonstrated in different studies with animal models, and these are documented in leading textbooks of neonatology $[6,12]$. The study by Basu also found a significant negative correlation between them and stages of HIE [13]. According to the study by Kavya, among the asphyxiated neonate's serum sodium, significantly lower calcium and glucose levels were observed in arterial cord blood and had a linear correlation with the severity of birth asphyxia and different stages of HIE.[14] The serum potassium and serum creatinine levels were higher in asphyxiated neonates and were correlating with the severity of asphyxia.

\section{Conclusion}

Perinatal asphyxia will result in neonatal hypoxia and tissue/organ injury. A variety of maternal, obstetric, and neonatal conditions predispose the fetus and newborn to asphyxia, which can occur before, during, or after birth. One of the most effective ways to reduce the risk is the vigilance of at-risk pregnancies and appropriate timely intervention. It is important to develop intrapartum fetal monitoring techniques and to assure that healthcare professionals have easier access even though many of these techniques is controversial and the ability to detect fetal compromise is often unknown. And the relationship between Perinatal asphyxia and blood sugar level is a significantly lower and negative relationship.

\section{References}

[1] Rai S, Bhatiyani KK, Kaur S. Effect of birth asphyxia on serum calcium and glucose level: A prospective study. Int $\mathrm{J}$ Sci Stud 2015; 3: 3-6.

[2] Bryce J, Boschi-Pinto C, Shibuya K, Black RE: WHO estimates of the causes of death in children. Lancet. 2005, 365: 1147-1152. 10.1016/S0140-6736(05)71877-8.

[3] Snyder EY, Cloherty JP (1998) perinatal asphyxia. In: Clohert JP, Stark AR (eds) Manual of neonatal care, 4th edn. Lippincott-Raven, Philadelphia, p 515-532.

[4] Singh M (2004) Care of the baby in the labor room. In: Singh $\mathrm{M}(\mathrm{ed})$ Care of the newborn, 6th edn. Sagar Publications, New Delhi, p 107.

[5] Jones MD Jr, Burd LI, Makowski EL, Meschia G, Battaglia FC (1975) cerebral metabolism in sheep: a comparative study of the adult, the lamb and the fetus. Am J Physiol 229: 235-239. 
[6] Volpe JJ (2000) Neurology of the newborn, 4th edn. WBSaunders, Philadelphia, pp 497-520.

[7] Vannucci RC, Nardis EE, Vannucci SJ (1980) cerebral metabolism during hypoglycemia and asphyxia in newborn dogs. BiolNeonate 38: 276-286.

[8] Erdag GC, Vitrinel A (2004) Can urinary uric acid/creatinine ratiobe used as an additional marker for neonatal asphyxia? Int Pediatr 19 (4): 217-219.

[9] Sarnat HB, Sarnat MS. Neonatal encephalopathy following fetal distress: a clinical and electroencephalographics study. Arch Neural. 1976; 33 (10): 696-705.

[10] Vannucci RC (1992) cerebral carbohydrate and energy metaboli smin perinatal hypoxic-ischemic brain damage. Brain Pathol 2: 229-234. doi: 10.1111/j.17503639.1992.tb00696.x

[11] Anwar M, Vannucci RC (1988) Autoradiographic determination of regional cerebral blood flow during hypoglycemia in newborn dogs. Pediatr Res 24: 41-45. doi: 10.1203/00006450-198807000-00011.

[12] Salhab WA, Wyckoff MH, Laptook AR, Perlman JM (2004) Initial hypoglycemia and neonatal brain injury in term infants with severe fetal acidemia. Pediatrics 114: 361-366. doi: 10.1542/ peds.114.2.36.

[13] Basu P, Som S, Das H, Choudhuri B. Electrolyte status in birth asphyxia. Indian J Pediatr. 2010; 77 (3): 259-262.

[14] Kavya, Rudrappa, S., \& Gopal, G. (2020). Study and correlate the severity of birth asphyxia with serum levels of glucose, uric acid and electrolytes in the cord blood of asphyxiated neonates. International Journal of Contemporary Pediatrics, 8 (1), 98 .

[15] Aundhakar, C. D., \& Bahatkar, K. (2021). Electrolyte status and plasma glucose levels in birth asphyxia: A case-control study. Journal of Medical Sciences (Taipei, Taiwan), 41 (1). 